Article

\title{
Some Exact Solutions to Non-Fourier Heat Equations with Substantial Derivative
}

\author{
Konstantin Zhukovsky *, Dmitrii Oskolkov and Nadezhda Gubina
}

Faculty of Physics, M.V. Lomonosov Moscow State University, Leninskie Gory, 119991 Moscow, Russia; oskolkov.di15@physics.msu.ru (D.O.); gubina_nadya@mail.ru (N.G.)

* Correspondence: zhukovsk@physics.msu.ru; Tel.: +7-495-9393177

Received: 10 April 2018; Accepted: 13 July 2018; Published: 18 July 2018

\begin{abstract}
One-dimensional equations of telegrapher's-type (TE) and Guyer-Krumhansl-type (GK-type) with substantial derivative considered and operational solutions to them are given. The role of the exponential differential operators is discussed. The examples of their action on some initial functions are explored. Proper solutions are constructed in the integral form and some examples are studied with solutions in elementary functions. A system of hyperbolic-type inhomogeneous differential equations (DE), describing non-Fourier heat transfer with substantial derivative thin films, is considered. Exact harmonic solutions to these equations are obtained for the Cauchy and the Dirichlet conditions. The application to the ballistic heat transport in thin films is studied; the ballistic properties are accounted for by the Knudsen number. Two-speed heat propagation process is demonstrated-fast evolution of the ballistic quasi-temperature component in low-dimensional systems is elucidated and compared with slow diffusive heat-exchange process. The comparative analysis of the obtained solutions is performed.
\end{abstract}

Keywords: exponential operator; differential operator; Guyer-Krumhansl equation; moving media; non-Fourier; heat conduction; Knudsen number

\section{Introduction}

Recent progress in technology and science has driven interest to studies of heat conduction beyond common Fourier law [1]: $\partial_{t} T=k \partial_{x}^{2} T$, where $k$ is the thermal diffusivity, $T$ is the temperature; Fourier law describes heat conduction in homogeneous matter at normal conditions well. New heat sources, such as lasers, microwaves etc, are employed in medicine, science and material processing for melting, welding, cutting, drilling, etc. Often heat source and treated media are in motion. Some of modern materials and media have one or two dimensions: ultra-thin films, layers and nano-wires. Highly inhomogeneous porous and multilayered media are also common in industry. First major deviations from Fourier law were found in liquid Helium and in some solid crystal dielectrics at low temperatures $<25^{\circ} \mathrm{K}[2-5]$. Proper phenomenon was called Second Sound [6]; its satisfactory qualitative mathematical description was proposed by Cattaneo and Vernotte [7], who supposed phonon heat transport in addition to Fourier heat diffusion. In terms of temperature it can be put as follows:

$$
\left(\tau \partial_{t}^{2}+\partial_{t}\right) T=k_{F} \nabla^{2} T
$$

where $k_{F}$ is the Fourier thermal diffusivity, $\tau=k_{F} / C^{2}$ is the relaxation time of the heat waves propagation, which relates the moments of the temperature change and of the respective heat flux change. Cattaneo-Vernotte constitution implies a phase lag between the heat flux vector and the temperature gradient; in addition to heat diffusion the temperature perturbation propagates in matter like damped sound-wave at finite speed $C=\sqrt{k_{F} / \tau}$. The relaxation time $\tau$ is associated with 
th phonon-phonon interaction time; at normal conditions it is very small: $\tau \approx 10^{-13} \mathrm{~s}$. The Cattaneo Equation (1) is the particular case of the telegrapher's equation (TE).

$$
\left(\partial_{t}^{2}+\varepsilon \partial_{t}\right) F(x, t)=\left(\alpha \partial_{x}^{2}+\kappa\right) F(x, t), \varepsilon, \alpha, \kappa=\text { const }
$$

which takes its name because it describes the electric signal propagation in long electric lines without radiation [8]. For heat conduction we have $\tau=1 / \varepsilon, k_{F}=\alpha / \varepsilon, \mu=\kappa / \varepsilon$ and $\mu=0$ in Equation (1); the source term, $\kappa \neq 0$, describes heat exchange with the environment of low excess temperatures. However, precise quantitative description of the Second Sound with Equation (1) was not successful. There are reports on non-Fourier heat transport in highly inhomogeneous matter even at normal conditions [9-17], in fuel droplets [18], in biomaterials [19], in energy saving and insulating materials [20], in graphene, nanofibers, carbon nanotubes, silicon wires, etc. [21-27]. Heat transport in these cases was found close to that described by Guyer and Krumhansl (GK) in $[28,29]$. In the one-dimensional case in terms of temperature alone it has the following form:

$$
\left(\partial_{t}^{2}+\varepsilon \partial_{t}-\delta \partial_{t, x, x}^{3}\right) T(x, t)=\left(\alpha \partial_{x}^{2}+\kappa\right) T(x, t), \alpha, \varepsilon, \delta, \kappa=\text { const. }
$$

In its pure form the Guyer-Krumhansl law has $\kappa=0$. The following formulation:

$$
\left(\tau \partial_{t}^{2}+\partial_{t}\right) T(x, t)=\left(D_{b} \partial_{t, x, x}^{3}+k_{F} \partial_{x}^{2}+\mu\right) T(x, t),
$$

involves the parameters $\tau=1 / \varepsilon, \mu=\kappa / \varepsilon, k_{F}=\alpha / \varepsilon$, and $D_{b}=\delta / \varepsilon$, where the latter, $k_{b}$, is the ballistic type heat conductivity. The parameters in the above Equations (3) and (4) have the following dimensions: $[\tau]=s,\left[k_{F}\right]=\frac{m^{2}}{s},\left[D_{b}\right]=m^{2},[\mu]=\frac{1}{s},[\kappa]=\frac{1}{s^{2}},[\alpha]=\frac{m^{2}}{s^{2}},[\delta]=\frac{m^{2}}{s}$, and $[\varepsilon]=\frac{1}{s}$.

Despite GK equation is usually associated with ballistic properties, which manifest when the characteristic system scale $L$ is comparable or less than the mean free path $l$ of the phonons $L<l$, contradictory opinions on it exist [30-32]. In particular, the term "ballistic" is questioned in the context of GK type heat conduction in macroscopic inhomogeneous materials at room temperature [10,11]. Moreover, more complicated system of equations was proposed for the ballistic heat transport in [30], while heat propagation in highly inhomogeneous materials was reported to be close to GK law $[9,11]$.

Solutions to Hyperbolic Heat Conduction Equation (HHCE) can be obtained both analytically and numerically [33-40], although numerical methods seem to be more commonly used [41-44]. Analytical study gives deeper insight in the problem; operational analytical approach and solutions to HHE were developed in [45-50]. This method easily handles also other linear DE of high order and fractional DE [51-58]. Use of the exponential differential operators, such as the heat operator $S=e^{\partial_{x}^{2}}$ [59] allows operational solution of GK-type Equation (4) as demonstrated in [47-50].

In what follows we will obtain some exact solutions to non-Fourier heat transfer in GK-type equation with the substantial derivative, where the speed of the media is constant, and will obtain some particular operational solutions to modified GK-type equation in this case. Moreover, we will study the important case of periodic initial conditions, which occur, for example, in polymer electrolyte fuel cells (PEFCs) [60-68], as well as in thin membranes and highly inhomogeneous porous periodic structures, in printed wired heating boards [69] etc. We will analyze the solutions for the heat transport, described by the system of inhomogeneous partial derivative equation, and use the Knudsen number to account for the ballistic conditions in 1- and 2-dimensional structures [70].

\section{Ballistic Heat Transport Equations with Substantial Derivative}

For ultra-thin films and wires neither pure GK nor Cattaneo laws describe exactly heat transport phenomena, but their combination with Fourier law; it forms the following inhomogeneous system 
of differential equations for the ballistic $\theta_{b}(x, t)$ and diffusive component $\theta_{d}(x, t)$ of the complete dimensionless quasi-temperature $\theta=\theta_{d}+\theta_{b}$ (see [30]):

$$
\begin{gathered}
\left\{\partial_{t}^{2}+2 \partial_{t}-\frac{10 K n_{b}^{2}}{3} \partial_{x}^{2}-3 K n_{b}^{2} \partial_{t, x, x}^{3}+1\right\} \theta_{b}(x, t)=0, \\
\left(\partial_{t}^{2}+\frac{K n_{b}^{2}}{K n_{d}^{2}} \partial_{t}-\frac{K n_{b}^{4}}{3 K n_{d}^{2}} \partial_{x}^{2}\right) \theta_{d}(x, t)=\left(\partial_{t}+\frac{K n_{b}^{2}}{K n_{d}^{2}}\right) \theta_{b}(x, t),
\end{gathered}
$$

where indices $b$ and $d$ stand for "ballistic" and "diffusive", respectively. The dimensionless quasi-temperature $\theta$ can be described as the non-dimensional energy, associated with the internal energy; the quantities $\theta_{d}$ and $\theta_{b}$ must therefore be understood to quasi-temperatures, defined as a measure of corresponding internal energy components $u_{d}$ and $u_{b}$, respectively to which they are related by the simple relations $\theta_{d}=u_{d} / c \theta_{b}=u_{b} / c$ (see [30] for details), where $c$ is the heat capacity.

It should be understood though, that the ballistic heat transport is effectively described by both contributions in $\theta=\theta_{d}+\theta_{b}, \theta_{d}(x, t)$ and $\theta_{b}(x, t)$ are distinguished for convenience. It is easy to see that the above Equations (5) and (6) are of GK-type (5) and of telegrapher's type (6); the inhomogeneous right hand side (r.h.s.) of Equation (6) is given by the solution of (5). In the above equations and in all following equations the space- and time-coordinates are obviously dimensionless as well as the proper coefficients in the equations are. The dimensionless form of equations for the temperature is useful at least because of the space-time temperature distribution is expressed in our work explicitly in terms of $x$ and $t$. Proper renormalization in SI units or else how is elementary (see, for example, [30] for details) and good, especially in view of heavy notations in the analytical solutions, which we will obtain in what follows.

Low-dimensional system is characterized by the dimensionless Knudsen number $K n=l / L$, which for the phonon heat transport describes the ratio of the free mean path of the phonons $l$ to the characteristic scale of the system $L$. Knudsen number usually arises in problems, where the scale of the system and the characteristic scale of the processes in it compare with each other, such as for gas flow in ultra-narrow channels [71], etc. In distinct ballistic case in (5), $K n=1$, we have $\alpha=3.333$, $\delta=3, \varepsilon=2, \kappa=-1$ in (3) and $k_{F}=5 / 3, D_{b}=3 / 2, \tau=1 / 2, \mu=-1 / 2$ in (4). We assume dimensionless equations here and in what follows. In the weak ballistic case, $K n=0.1$, we get $\alpha=0.03333, \delta=0.03, \varepsilon=2$, $\kappa=-1$ in (3) and $k_{F}=0.01666, k_{b}=0.015, \tau=1 / 2, \mu=-1 / 2$ in (4). In the distinct ballistic case, $K n=1$, all heat transport terms in GK-type equation contribute more or less equally, in the weak ballistic case, $K n=0.1$, the Cattaneo wave-term prevails. The inhomogeneous system of PDE (5) and (6) for ballistic heat transport thin film was studied numerically in [30]; based on the periodic analytical solutions to GK-type equation [72] some solutions to (5) and (6) were obtained in [73,74].

Above Equations (5) and (6), as well as Equations (2)-(4), describe non-Fourier heat transport in stationary media. In the case when the observer moves relatively to the media with constant speed $\vec{v}$, the substantial derivative $D / D t=\partial / \partial t+\vec{v} \vec{\nabla}$ should be used instead of the time-derivative $\partial / \partial t$ to describe the variation of quantity $F$ along a path. Generally speaking, both the material equations and the energy, mass and momentum conservation equations in case of moving observer should be considered with account for the substantial derivative, resulting in a proper heat conduction equation for the temperature alone. This is relatively simple in the case of Fourier heat diffusion and even for relativistic heat equation $[75,76]$, some more complicated for GK-type equation. While not intending to rigorously derive a set of equations for the heat transport in case of moving observer, accounting for the relativistic heat flux, the energy balance, etc., we will solve the extended form of the known GK-type equation with the simple substantial derivative. Derivation of a physically comprehensive one-dimensional analogue of GK-equation in case of moving observer remains beyond the scope 
of the present study. The following modified form of GK-type equation arises upon the use of the substantial time derivative:

$$
\left(\partial_{t}^{2}+\varepsilon \partial_{t}+2 v \partial_{t, x}^{2}-\delta \partial_{t, x, x}^{3}\right) T(x, t)=\left(\left(\alpha-v^{2}\right) \partial_{x}^{2}-\varepsilon v \partial_{x}+\delta v \partial_{x}^{3}+\kappa\right) T(x, t) .
$$

This simply presumes that the temperature as a function of time is recorded by a floating instrument in a flow, such as a weather balloon in meteorology and oceanography, which implies the substantial derivative along the pathline traveled. With this said, we notice that the above Equation (7) differs from GK-type Equation (4) by some additional terms: the second mixed time-space derivative in the left hand side (1.h.s.) and the third- and first-order space-derivatives in the r.h.s. We need to emphasize that this equation is related to a rigid domain meanwhile the observer is moving with speed $\vec{v}$. Moreover, the coefficient for the second-order space-derivative in the r.h.s. now contains the speed $v$, which can compensate $\alpha$, eliminating the second-order space-derivative, if $v=\sqrt{\alpha}$, or invert the sign of this term, if $v>\sqrt{\alpha}$. Telegrapher's equation accordingly modifies as follows:

$$
\left(\partial_{t}^{2}+\varepsilon \partial_{t}+2 v \partial_{t, x}^{2}\right) T(x, t)=\left(\left(\alpha-v^{2}\right) \partial_{x}^{2}-\varepsilon v \partial_{x}+\kappa\right) T(x, t) .
$$

Differently from GK-type equation with substantial derivative (7), TE (8) has only second-order differential operators; both GK-type and TE can be solved by the operational method, employing exponential differential operators $e^{\hat{D}}$ and involving the heat operator $\hat{S}=e^{\partial_{x}^{2}}$ [59]. The operational solution to the third-order PDE (7) apparently involves exponential differential operator of the third order $e^{a \partial_{x}^{3}}$. However, in what follows we will demonstrate as this solution effectively reduces to the shift of the solution in a stationary media. Considering thin films, we have obvious relations between the coefficients in (7) and (5): $\alpha_{b} \leftrightarrow 10 K n_{b}^{2} / 3, \varepsilon_{b} \leftrightarrow 2, \delta \leftrightarrow 3 K n_{b}^{2}, \kappa_{b} \leftrightarrow-1$, while for Equation (6) we see that $\alpha_{d} \leftrightarrow K n_{b}^{4} / 3 K n_{d}^{2}, \varepsilon_{d} \leftrightarrow K n_{b}^{2} / K n_{d}^{2}$. For heat transport in moving thin film, the system of $\mathrm{DE}(5)$ and (6) for the complete quasi-temperature $\theta=\theta_{d}+\theta_{b}$ takes the following form:

$$
\begin{gathered}
\left(\partial_{t}^{2}+2 \partial_{t}+2 v \partial_{t, x}^{2}-\left(\frac{10}{3} K n_{b}^{2}-v^{2}\right) \partial_{x}^{2}-3 K n_{b}^{2} \partial_{t, x, x}^{3}+2 v \partial_{x}-3 v K n_{b}^{2} \partial_{x}^{3}+1\right) \theta_{b}(x, t)=0, \\
\left(\partial_{t}^{2}+\frac{K n_{b}^{2}}{K n_{d}^{2}} \partial_{t}+2 v \partial_{t, x}^{2}-\left(\frac{K n_{b}^{4}}{3 K n_{d}^{2}}-v^{2}\right) \partial_{x}^{2}+v \frac{K n_{b}^{2}}{K n_{d}^{2}} \partial_{x}\right) \theta_{d}(x, t)=\left(\partial_{t}+v \partial_{x}+\frac{K n_{b}^{2}}{K n_{d}^{2}}\right) \theta_{b}(x, t) .
\end{gathered}
$$

In what follows we will approach the GK-type equation with substantial derivative (7) operationally, and will also provide exact analytical harmonic solution to the system of PDE (9) and (10), describing ballistic heat transfer with substantial derivative.

\section{Operational Approach to Transport Equations}

The above Equations (7) and (8) are the particular cases of the following DE with the coordinate-dependent operators $\hat{\varepsilon}(x)$ and $\hat{D}(x)$ :

$$
\left(\partial_{t}^{2}+\hat{\varepsilon}(x) \partial_{t}\right) F(x, t)=\hat{D}(x) F(x, t)
$$

which can be solved using the operational method. Equation (11) models a broad spectrum of physical phenomena. It becomes telegrapher's equation for $\hat{D}(x)=\alpha \partial_{x}^{2}+\kappa$ and constant $\varepsilon$ term and further reduces to Cattaneo heat equation for $\hat{D}(x)=\partial_{x}^{2}$; GK-type equation appears when $\hat{\varepsilon}=\varepsilon-\delta \partial_{x}^{2}$. Some of these equations were studied in [45-50]. Other second-order PDE and fractional DE were explored with the help of the operational approach in [51-58]. The formal converging particular operational solution to (11) reads as follows:

$$
F(x, t)=e^{-\frac{t \hat{\varepsilon}(x)}{2}} e^{-\frac{t}{2} \sqrt{\hat{\varepsilon}^{2}(x)+4 \hat{D}(x)}} C(x),
$$


where $C(x)$ can be obtained from the initial condition $F(x, 0)=f(x)$. The particular form of the initial function will be chosen below. The other branch of the solution contains the positive argument in the exponential, $e^{\frac{t}{2}} \sqrt{\hat{\varepsilon}^{2}(x)+4 \hat{D}(x)}$. No Laplace transforms exist for it. However, symmetry with respect to inversion, $t \rightarrow-t, \varepsilon \rightarrow-\varepsilon, \delta \rightarrow-\delta, v \rightarrow-v$, allows writing the other solution to Equation (7), based on the one we obtain with the help of the Laplace transforms

$$
e^{-t \sqrt{V}}=\frac{t}{2 \sqrt{\pi}} \int_{0}^{\infty} \frac{d \xi}{\xi \sqrt{\xi}} e^{-\frac{t^{2}}{4 \xi}-\xi V}, t>0
$$

The solution has the following integral form, provided it converges:

$$
F(x, t)=e^{-\frac{t}{2} \hat{\varepsilon}(x)} \frac{t}{4 \sqrt{\pi}} \int_{0}^{\infty} \frac{d \xi}{\xi \sqrt{\xi}} e^{-\frac{t^{2}}{16 \xi}} e^{-\xi^{\xi} \hat{\varepsilon}^{2}(x)} e^{-4 \xi \hat{\mathcal{D}}(x)} f(x) .
$$

The ability to perform analytical integration in (14) depends on the explicit form of the operators $\hat{\varepsilon}, \hat{D}$ and of the initial function $f(x)$; the numerical calculation can be done though. Accounting for the explicit form of the operators $\hat{D}=\left(\alpha-v^{2}\right) \partial_{x}^{2}-\varepsilon v \partial_{x}+\delta v \partial_{x}^{3}+\kappa$ and $\hat{\varepsilon}=\varepsilon+2 v \partial_{x}-\delta \partial_{x}^{2}$ in Equation (7), we obtain from (14) the following integral:

$$
F(x, t)=e^{-\frac{t}{2}\left(\varepsilon+2 v \partial_{x}\right)} \frac{t}{4 \sqrt{\pi}} \int_{0}^{\infty} \frac{d \xi}{\xi \sqrt{\zeta}} e^{-\frac{t^{2}}{16 \xi}-\xi\left(\varepsilon^{2}+4 \kappa\right)} e^{\left(\frac{t}{2} \delta+2 \xi(\varepsilon \delta-2 \alpha)\right) \partial_{x}^{2}} e^{-\xi^{2} \partial_{x}^{4}} f(x),
$$

which benefits from the use of the operational identity for $\hat{p}=\sqrt{a} \hat{D}, \hat{D}=\partial^{2}$ (see $\left.[59,77]\right)$ :

$$
e^{\hat{p}^{2}}=\frac{1}{\sqrt{\pi}} \int_{-\infty}^{\infty} \exp \left(-\xi^{2}+2 \xi \hat{p}\right) d \xi
$$

and yields

$$
e^{a \hat{D}^{2}} f(x)=\frac{1}{\sqrt{\pi}} \int_{-\infty}^{\infty} \exp \left(-\xi^{2}+2 \xi \sqrt{a} \hat{D}\right) f(x) d \xi .
$$

Applying formula (17) to the fourth-order exponential differential operator $e^{-\xi^{2} \partial_{x}^{4}} f(x)=\int_{-\infty}^{\infty} e^{-\zeta^{2}+2 i \zeta \delta \sqrt{\zeta} \partial_{x}^{2}} f(x) d \zeta / \sqrt{\pi}$, we get the heat operator [59] $\hat{S}=e^{\nu \partial_{x}^{2}}$; collecting the second-order derivative terms in the exponential, we get $\int_{-\infty}^{\infty} e^{-\zeta^{2}+((t \delta / 2)-4 \xi \alpha+2 \xi \varepsilon \delta+2 i \zeta \delta \sqrt{\zeta}) \partial_{x}^{2}} f(x) d \zeta$. This yields the following particular bounded solution to GK-type heat equation with substantial derivative (7):

$$
F(x, t)=\frac{e^{-\frac{t}{2} \varepsilon} t}{4 \pi} \hat{\Theta} \int_{0}^{\infty} \frac{d \xi}{\xi \sqrt{\xi}} e^{-\frac{t^{2}}{16 \xi}-\tilde{\zeta}\left(\varepsilon^{2}+4 \kappa\right)} \int_{-\infty}^{\infty} e^{-\zeta^{2}} \hat{S} f(x) d \zeta,
$$

which involves the shift operator $\hat{\Theta}=e^{y \partial x}$, where $y=-v t$, and the heat operator $\hat{S}=e^{\eta \partial_{x}^{2}}$, where $\eta=2 i \zeta \sqrt{\xi} \delta-\xi 2(2 \alpha+\varepsilon \delta)+t \delta / 2$. The shift operator produces the translation along $x e^{y\left(\partial_{x}+\alpha\right)} f(x)=e^{y \alpha} f(x+y)$ and the heat operator produces Gauss transforms (17) and operational relations [59], so that the solution with account for the motion contains the translation $x-v t$ of the stationary solution.

Let us consider the initial polynomial function $f(x)=\sum_{k} x^{k}$. The particular solution (18) to GK-type equation with substantial derivative (7) arises upon the application of the operational rule

$$
\hat{S} e^{\gamma x} x^{k}=e^{\eta \partial_{x}^{2}} x^{k} e^{\gamma x}=e^{\gamma x+\gamma^{2} v} H_{k}(x+2 \gamma v, v), k \in \text { Integers, } \gamma \in \text { Reals, }
$$


which is easy to prove $[57,58]$. For $\gamma=0$ we immediately obtain the following integral:

$$
\left.F(x, t)\right|_{f(x)=\Sigma_{k} x^{k}}=\sum_{k} \frac{t e^{-\frac{t}{2} \varepsilon}}{4 \pi} \int_{0}^{\infty} \frac{d \xi}{\xi \sqrt{\xi}} e^{-\frac{t^{2}}{16 \xi}-\xi\left(\varepsilon^{2}+4 \kappa\right)} \int_{-\infty}^{\infty} e^{-\zeta^{2}} H_{k}(x-v t, \eta) d \zeta,
$$

where

$$
\eta=2 \xi \varepsilon \delta-4 \xi \alpha+t \delta / 2+i \zeta 2 \sqrt{\xi} \delta .
$$

The above integral can be taken in elementary functions if we account for the explicit form of the Hermite polynomials:

$$
H_{k}(x-v t, \eta)=k ! \sum_{r=0}^{[k / 2]} \frac{(x-v t)^{k-2 r} \eta^{r}}{(k-2 r) ! r !}=(-i)^{k} \eta^{\frac{k}{2}} H_{k}\left(\frac{i(x-v t)}{2 \sqrt{\eta}}\right) .
$$

For example, for $f(x)=x^{2}$ the solution reads as follows:

$$
\left.F(x, t)\right|_{f(x)=x^{2}}=e^{-\frac{t}{2}(\sqrt{V}+\varepsilon)}\left((x-v t)^{2}+t \delta+\frac{t}{\sqrt{V}}(\delta \varepsilon-2 \alpha)\right), V=\varepsilon^{2}+4 \kappa^{2} .
$$

More general case of the exponential-polynomial function $f(x)=x^{k} e^{\gamma x}$ is cumbersome and we omit proper expressions for conciseness. However, we have performed the integration explicitly in Wolfram Mathematica program and below we give the example of the solution for $F(x, 0)=x^{2} e^{-x}$ :

$$
\begin{aligned}
& \left.F(x, t)\right|_{f(x)=x^{2} e^{-x}}=e^{-x-\frac{t}{2}(\sqrt{r}-2 v-\delta+\varepsilon)}\left(x^{2}+\frac{t}{r^{3 / 2}}(a+b x)+\frac{t^{2}}{r^{3 / 2}} c\right), \\
& r=4 \alpha+\delta^{2}-2 \delta \varepsilon+\varepsilon^{2}+4 \kappa \\
& a=\sqrt{r} \delta\left(4 \alpha+(\varepsilon-\delta)^{2}\right)+\delta^{2}\left(-6 \alpha-\delta^{2}-3 \varepsilon(\varepsilon-\delta)\right)+ \\
& \varepsilon^{2}(-2 \alpha+\delta \varepsilon)-4 \kappa\left(2 \alpha+\sqrt{r} \delta-3 \delta^{2}+\delta \varepsilon\right) \\
& b=2 r\left(2 \alpha+\delta^{2}-\sqrt{r} v-\delta(\sqrt{r}+\varepsilon)\right) \\
& c=r^{3 / 2} v^{2}-2 r v d+2 p+\delta^{2} \Delta \\
& d=2 \alpha+\delta(-\sqrt{r}+\delta-\varepsilon) \\
& p=2 \alpha^{2}(\sqrt{r}-4 \delta)-2 \alpha \delta\left(3 \delta^{2}+\sqrt{r} \varepsilon+\varepsilon^{2}-2 \delta(\sqrt{r}+2 \varepsilon)+4 \kappa\right), \\
& \Delta=\sqrt{r}(\varepsilon-\delta)^{2}+(\varepsilon-\delta)^{3}+2 \kappa(\sqrt{r}+2(\varepsilon-\delta)) .
\end{aligned}
$$

Evidently, all the values in the above expressions are dimensionless as well as the coordinate $x$ and the time $t$.

In the harmonic ansatz the evolution of the initial function $f(x)=\exp (\operatorname{in} x)$ can be easily obtained from (18) as follows:

$$
\left.F(x, t)\right|_{f(x)=e^{i n x}}=\exp \left(i n(x-v t)-\frac{t}{2}\left(\bar{\varepsilon}+\sqrt{\bar{\varepsilon}^{2}+4\left(\kappa^{2}-\alpha n^{2}\right)}\right)\right), \bar{\varepsilon}=\varepsilon+n^{2} \delta .
$$

Other solutions and their detailed study will be performed in forthcoming publications. All the above solutions to equations with substantial derivative contain the shift, $x-v t$, respectively to the solution in a stationary media, which depends on $x$. Further examples can be easily considered with the help of the operational approach. In the following chapter we will explore in details the particular case of the harmonic solution. Evidently, it can be easily generalized for any periodic solution, expandable in Fourier series. Similarly, the Fourier integral transforms technique apply.

\section{Exact Periodic Solutions to GK-Type Equation with Substantial Derivative}

An exact harmonic solution $\propto e^{i n x}$ for the inhomogeneous system of PDE (9) and (10) can be obtained straight from the operational solution (18) (see (25)), or by separating the variables: 
$T(x, t)=X(x) y(t)$. GK-type equation with substantial derivative (7) in the harmonic ansatz, $T(x, t)=e^{i n x} y(t) \equiv F(x, t)$ reduces to the following ordinary differential equation for $y(t)$ :

$$
y^{\prime \prime}(t)+\varepsilon_{e f f} y^{\prime}(t)+\lambda_{e f f} y(t)=0,
$$

where $\varepsilon_{e f f}=\bar{\varepsilon}+2 v i n, \bar{\varepsilon}=\varepsilon+n^{2} \delta$, and $\lambda_{\text {eff }}=\left(\alpha-v^{2}\right) n^{2}+i v n \bar{\varepsilon}-\kappa$. The function $F(x, t) \equiv T(x, t)$ is introduced here for clarity of notations. The exact solution to GK equation with substantial derivative (7) then easily follows from the solution to (26):

$$
\begin{gathered}
F(x, t)=e^{i n x} y(t), y(t)=e^{-\frac{t}{2} \varepsilon_{e f f}}\left(C_{1} e^{-\frac{t}{2} \sqrt{u}}+C_{2} e^{\frac{t}{2} \sqrt{u}}\right), \\
U=\varepsilon_{e f f}^{2}-4 \lambda_{e f f}=\bar{\varepsilon}^{2}+4\left(\kappa-\alpha n^{2}\right),
\end{gathered}
$$

where the constants $C_{1}, C_{2}$ are determined either from the initial or boundary conditions. According to the theory of separation of variables, the whole solution has to satisfy initial or boundary conditions. Evidently, telegrapher's equation with substantial derivative in the harmonic ansatz similarly reads

$$
y^{\prime \prime}(t)+\widetilde{\varepsilon} y^{\prime}(t)+\widetilde{\lambda} y(t)=0,
$$

and differs from (26) by the substitutions $\varepsilon_{e f f} \rightarrow \widetilde{\varepsilon}=\varepsilon+2 v i n, \quad \bar{\varepsilon} \rightarrow \varepsilon$, $\lambda_{\text {eff }} \rightarrow \widetilde{\lambda}=\left(\alpha-v^{2}\right) n^{2}+i v n \varepsilon-\kappa$. It's solution $y(t)$ is evidently (27), where $\delta=0$.

Consider, for example, the Cauchy initial conditions

$$
F(x, 0)=A e^{i n x}, \partial_{t} F(x, 0)=B e^{i n x} .
$$

In the stationary case, $v=0$, the Cauchy conditions (37), where initially $\frac{\partial \theta(x, t)}{\partial t}=0$, have the meaning of zero heat flux $q$ if $B=0$ for both ballistic and diffusive components of quasi-temperature [30]. If $v \neq 0$, then the dimensionless equation $\theta^{\prime}{ }_{t}+v \theta^{\prime}{ }_{x}+q=0$ describes the one-dimensional energy balance. The initially zero heat flux $q$ then corresponds to $B=-i n v A$.

Then, GK-type Equation (7) with substantial derivative in the harmonic ansatz has the solution (27), satisfying ODE (26), where the coefficients $C_{1}, C_{2}$ are determined from the Cauchy conditions (29): $C_{1}+C_{2}=A$ and $-C_{1}\left(\varepsilon_{e f f}+\sqrt{U}\right)-C_{2}\left(\varepsilon_{e f f}-\sqrt{U}\right)=2 B$, and read as follows:

$$
C_{1}=\frac{A}{2}-\frac{B+A \varepsilon_{e f f} / 2}{\sqrt{U}}, C_{2}=\frac{A}{2}+\frac{B+A \varepsilon_{e f f} / 2}{\sqrt{U}} .
$$

TE with substantial derivative (8) has the harmonic solution, whose time-dependent part $y(t)$ satisfies ODE (28):

$$
F(x, t)=e^{i n x} y(t), y(t)=e^{-\frac{t}{2} \widetilde{\varepsilon}}\left(B_{1} e^{-\frac{t}{2} \sqrt{u}}+B_{2} e^{\frac{t}{2} \sqrt{u}}\right), u=\widetilde{\varepsilon}^{2}-4 \widetilde{\lambda}=\varepsilon^{2}+4\left(\kappa-\alpha n^{2}\right),
$$

where the coefficients $B_{1}, B_{2}$ are obtained from the Cauchy initial conditions (29):

$$
B_{1}=\frac{A}{2}-\frac{B+A \widetilde{\varepsilon} / 2}{\sqrt{u}}, B_{2}=\frac{A}{2}+\frac{B+A \widetilde{\varepsilon} / 2}{\sqrt{u}} .
$$

Now, let us consider the Dirichlet boundary conditions in the moments of time $t=0$ and $t=T$ :

$$
F(x, 0)=A e^{i n x}, F(x, T)=G e^{i n x} .
$$


The harmonic solutions (27) and (31) satisfy respectively GK Equation (7) and telegrapher's Equation (8) with substantial derivative with the coefficients, determined by Equation (33):

$$
C_{1}=-e^{\frac{T}{2} \varepsilon_{e f f}} \frac{A e^{-\frac{T}{2}\left(\varepsilon_{e f f}-\sqrt{U}\right)}-G}{e^{-\frac{T}{2} \sqrt{U}}-e^{\frac{T}{2} \sqrt{U}}}, C_{2}=A-C_{1},
$$

and

$$
B_{1}=-e^{\frac{T}{2} \widetilde{\varepsilon}} \frac{A e^{-\frac{T}{2}(\widetilde{\varepsilon}-\sqrt{u})}-G}{e^{-\frac{T}{2} \sqrt{u}}-e^{\frac{T}{2} \sqrt{u}}}, B_{2}=A-B_{1} .
$$

Similarly, we can consider evolution of any function, expandable in Fourier series.

Note, that for some values of the coefficients $\alpha$ and $\delta$ the quantities $U$ and $u$ can assume negative values. Albeit it is not very obvious in the form of the harmonic solution (27) to GK-type equation with substantial derivative (26) and the solution (31) to TE with substantial derivative (28), these solutions remains real at any moment of time for a real initial function, because of the complex exponential is compensated by proper complex parts in the coefficients $C_{1,2}$ and $B_{1,2}$.

\section{Exact Periodic Solutions to Ballistic Heat Transport in Thin Films}

Ballistic heat transport in thin films is described by the system of PDE (9) and (10). In order to obtain the exact solution to this problem we reduced GK-type Equation (9) in the harmonic ansatz to (26) and solved it (see (27)); the result naturally involves the particular solution (25). This solution now constitutes the r.h.s. of Equation (10) for $\theta_{d}$. Consider the Cauchy initial problem for $\theta_{b}$ :

$$
\theta_{b}(x, 0)=A e^{i n x}, \partial_{t} \theta_{b}(x, 0)=B e^{i n x},
$$

and the Cauchy conditions for $\theta_{d}$ :

$$
\theta_{d}(x, 0)=V e^{i n x}, \partial_{t} \theta_{d}(x, 0)=W e^{i n x}, V, W=\text { const. }
$$

The quasi-temperature component $\theta_{b}(x, t)$ is in fact given by Equation (27):

$$
\theta_{b}(x, t)=e^{i n x}\left(C_{1} e^{-\frac{t}{2} E_{1}}+C_{2} e^{-\frac{t}{2} E_{2}}\right), E_{1}=\left(\varepsilon_{e f f}+\sqrt{U}\right), E_{2}=\left(\varepsilon_{e f f}-\sqrt{U}\right),
$$

where $\varepsilon_{\text {eff }}=\bar{\varepsilon}+2 i v n, U=\bar{\varepsilon}^{2}+4\left(\kappa-\alpha_{b} n^{2}\right), \bar{\varepsilon}=\varepsilon_{b}+n^{2} \delta, \alpha_{b}=10 K n_{b}^{2} / 3, \varepsilon_{b}=2, \delta=3 K n_{b}^{2}, \kappa_{b}=-1$, $v$ is the speed of the media; for the Cauchy initial conditions (36) (see (29) with $F \equiv \theta_{b}$ ) the coefficients $C_{1,2}$ are given by Equation (30). The solution (38) for $\theta_{b}(x, t)$ contributes to the r.h.s. of the telegrapher's Equation (6) for the component $\theta_{d}(x, t)$. The exponential differential operators $e^{\hat{D}(x)}$ do not bring new harmonics to the initial content, $\theta_{d}(x, t)=\Theta_{d}(t) e^{i n x}$, and Equation (6) reduces to the following inhomogeneous ODE for $\Theta_{d}(t)$ :

$$
\left(\frac{d^{2}}{d t^{2}}+\widetilde{\varepsilon}_{d} \frac{d}{d t}+\widetilde{\lambda}_{d}\right) \Theta_{d}(t)=+P e^{-\frac{t}{2} E_{1}}+Q e^{-\frac{t}{2} E_{2}}
$$

where

$$
\begin{gathered}
\widetilde{\varepsilon}_{d}=\varepsilon_{d}+i 2 v n, \widetilde{\lambda}_{d}=\left(\alpha_{d}-v^{2}\right) n^{2}+i v n \varepsilon_{d}, \varepsilon_{d}=\frac{K n_{b}^{2}}{K n_{d}^{2}}, \alpha_{d}=\frac{K n_{b}^{4}}{3 K n_{d}^{2}} \\
P=C_{1}\left(\varepsilon_{d}-\frac{\bar{\varepsilon}+\sqrt{U}}{2}+i v n\right), Q=C_{2}\left(\varepsilon_{d}-\frac{\bar{\varepsilon}-\sqrt{U}}{2}+i v n\right)
\end{gathered}
$$


where $C_{1,2}$ are given by Equation (30). The above Equation (39) for $\Theta_{d}(t)$ possesses analytical solution, which, in turn, yields the evolving in time harmonic solution for the diffusive quasi-temperature component $\theta_{d}(x, t)$ in the following form:

$$
\begin{aligned}
\theta_{d}(x, t) & =e^{i n x}\left(4\left(\frac{P}{S} e^{-\frac{t}{2} E_{1}}+\frac{Q}{L} e^{-\frac{t}{2} E_{2}}\right)+D_{1} e^{-\frac{t}{2}\left(\widetilde{\varepsilon}_{d}+R\right)}+D_{2} e^{-\frac{t}{2}\left(\widetilde{\varepsilon}_{d}-R\right)}\right), \\
R & =\sqrt{\widetilde{\varepsilon}_{d}^{2}-4 \widetilde{\lambda}_{d}}=\sqrt{\varepsilon_{d}^{2}-4 n^{2} \alpha_{d}} \\
S & =E_{1}^{2}+4 \widetilde{\lambda}_{d}-2 E_{1} \widetilde{\varepsilon}_{d}=\left(\bar{\varepsilon}-\varepsilon_{d}+\sqrt{U}\right)^{2}+4 \alpha_{d} n^{2}-\varepsilon_{d^{\prime}}^{2} \\
L & =E_{2}^{2}+4 \widetilde{\lambda}_{d}-2 E_{2} \widetilde{\varepsilon}_{d}=\left(\bar{\varepsilon}-\varepsilon_{d}-\sqrt{U}\right)^{2}+4 \alpha_{d} n^{2}-\varepsilon_{d^{\prime}}^{2}
\end{aligned}
$$

where the constants $D_{1,2}$ are determined from the Cauchy initial conditions (37) for $\theta_{d}(x, t)$ :

$$
\begin{aligned}
& D_{1}=\frac{V}{2}\left(1-\frac{\widetilde{\varepsilon}_{d}}{R}\right)-\frac{1}{R}\left(W+\frac{2 P}{S}\left(\bar{\varepsilon}-\varepsilon_{d}+\sqrt{U}+R\right)+\frac{2 Q}{L}\left(\bar{\varepsilon}-\varepsilon_{d}-\sqrt{U}+R\right)\right), \\
& D_{2}=\frac{V}{2}\left(1+\frac{\widetilde{\varepsilon}_{d}}{R}\right)+\frac{1}{R}\left(W+\frac{2 P}{S}\left(\bar{\varepsilon}-\varepsilon_{d}+\sqrt{U}-R\right)+\frac{2 Q}{L}\left(\bar{\varepsilon}-\varepsilon_{d}-\sqrt{U}-R\right)\right) .
\end{aligned}
$$

The exact analytical harmonic solutions for the $\theta_{b}(x, t)$ is given by (38) and (30), and for $\theta_{d}(x, t)$, it is given by (41)-(44); their sum represents the complete solution for the ballistic heat transfer in thin films [30] with the Cauchy initial conditions (36), (37), valid for arbitrary values of the Knudsen numbers $K n_{d}, K n_{b}$. Note, that a real initial distribution of quasi-temperature $\theta(x, 0)$ evolves in real domain even for imaginary exponentials in the solutions due to complex coefficients $C_{1}, C_{2}$ and $D_{1}, D_{2}$.

Now let us consider the Dirichlet conditions at the boundaries

$$
\theta_{b}(x, 0)=A e^{i n x}, \theta_{b}(x, T)=G e^{i n x}, \theta_{d}(x, 0)=V e^{i n x}, \theta_{d}(x, T)=W e^{i n x},
$$

where $A, G, V, W=$ const (see also Equation (33)). The evolution of $\theta_{b}(x, t)$ is given by Equation (38), where $C_{1,2}$ are set by Equation (34). The evolution of the counterpart $\theta_{d}(x, t)$ is governed by Equations (39) and (40). The r.h.s. of Equation (39) is set by Equation (38) for the ballistic component $\theta_{b}(x, t)$, where $C_{1,2}$ are given by (34). The explicit solution $\theta_{d}(x, t)$ to inhomogeneous DE (39) is possible, although it is much more cumbersome than in the Cauchy case:

$$
\theta_{d}(x, t)=e^{i n x}\left(\frac{b_{2}}{w_{1}} e^{-E_{2} t}+\frac{b_{1}}{w_{2}} e^{-E_{1} t}+\frac{q_{1}}{w_{1} w_{2}} e^{-\frac{1}{2} t\left(\varepsilon_{d}+R\right)}-\frac{q_{2}}{w_{1} w_{2}} e^{-\frac{1}{2} t\left(\varepsilon_{d}-R\right)}\right),
$$

where

$$
\begin{gathered}
w_{1}=E_{2}^{2}-E_{2} \widetilde{\varepsilon}_{d}+\widetilde{\lambda}_{d}, w_{2}=E_{1}^{2}-E_{1} \widetilde{\varepsilon}_{d}+\widetilde{\lambda}_{d} \\
q_{1}=\frac{1}{e^{R T}-1}\left(e^{-E_{2} T} w_{1} b_{1} d_{2}+e^{-E_{2} T} b_{2} d_{2} w_{2}+d_{1} w_{1} w_{2}\right), \\
q_{2}=\frac{1}{e^{R T}-1}\left(e^{-E_{2} T}\left(\widetilde{\lambda}_{d}-w_{2}\right)\left(b_{2} d_{6}-w_{1} d_{4}\right)+e^{-E_{1} T}\left(w_{1}\left(\widetilde{\lambda}_{d} d_{3}-b_{1} d_{5}\right)+\widetilde{\lambda}_{d} b_{2} d_{7}\right)\right), \\
b_{1}=C_{1}\left(\widetilde{\varepsilon}_{d}-\frac{\varepsilon_{e f f}+\sqrt{U}}{2}+i v n\right), b_{2}=C_{2}\left(\widetilde{\varepsilon}_{d}-\frac{\varepsilon_{e f f}-\sqrt{U}}{2}+i v n\right), d_{1}=V e^{R T}-W e^{\frac{T}{2}\left(\widetilde{\varepsilon}_{d}+R\right)}, \\
d_{2}=e^{\frac{T}{2}\left(\widetilde{\varepsilon}_{d}+R\right)}-e^{T\left(E_{1}+R\right)}, d_{3}=V e^{E_{1} T}-W e^{\left(2 E_{1}+\widetilde{\varepsilon}_{d}+R\right) \frac{T}{2}}, d_{4}=V e^{E_{2} T}-W e^{\left(2 E_{2}+\widetilde{\varepsilon}_{d}+R\right) \frac{T}{2}} \\
d_{5}=e^{E_{1} T}-e^{\left(\widetilde{\varepsilon}_{d}+R\right) \frac{T}{2}}, d_{6}=e^{E_{2} T}-e^{\left(\widetilde{\varepsilon}_{d}+R\right) \frac{T}{2}}, d_{7}=-e^{E_{1} T}+e^{\left(\widetilde{\varepsilon}_{d}+R+2\left(E_{1}-E_{2}\right)\right) \frac{T}{2}} .
\end{gathered}
$$

The solution for $\theta_{d}(x, t)$ in the form (46), (47) explicitly involves the Dirichlet conditions and together with the solution for $\theta_{b}(x, t)$, given in the Dirichlet case by (38) and (34), they describe the evolution of the complete harmonic quasi-temperature $\theta=\theta_{d}+\theta_{b}$. 
Let us now consider some examples of the harmonic solutions to GK-type heat equation with substantial derivative. Consider, for example, the Cauchy problem with initial conditions $\theta_{b}(x, 0)=\theta_{d}(x, 0)=e^{i x}, \partial_{t} \theta_{b}(x, 0)=\partial_{t} \theta_{d}(x, 0)=0$. Let's first consider $K n_{b}=K n_{d}=1$. Proper solutions for $\theta_{b}(x, t), \theta_{d}(x, t)$ and $\theta(x, t)$ in the stationary media are presented in Figure 1.

It can be seen in the top left plot in Figure 1 as the ballistic component $\theta_{b}(x, t)$ rapidly relaxes to the stationary state. The diffusive component $\theta_{d}(x, t)$ behaves similarly, but the relaxation process takes much more time. The behavior of ballistic and diffusive components in the domain is more distinct for $K n<1$. The complete quasi-temperature $\theta(x, t)$ (see bottom plot in Figure 1) follows the behavior of both the ballistic $\theta_{b}(x, t)$ and diffusive $\theta_{d}(x, t)$ constituents. However, the latter, being the solution of Equation (39), is strongly influenced by the solution (38) for the ballistic component $\theta_{b}(x, t)$, which sets the inhomogeneous part in Equation (39). Thus, the evolution of the quasi-temperature $\theta(x, t)$ in thin films has two speeds: the ballistic constituent $\theta_{b}(x, t)$ develops rapidly and sets the r.h.s. in Equation (39) for the diffusive constituent $\theta_{d}(x, t)$. The evolution of $\theta_{d}(x, t)$ is much slower: after the first instances it proceeds under the influence of already relaxed true ballistic constituent $\theta_{b}(x, t)$.
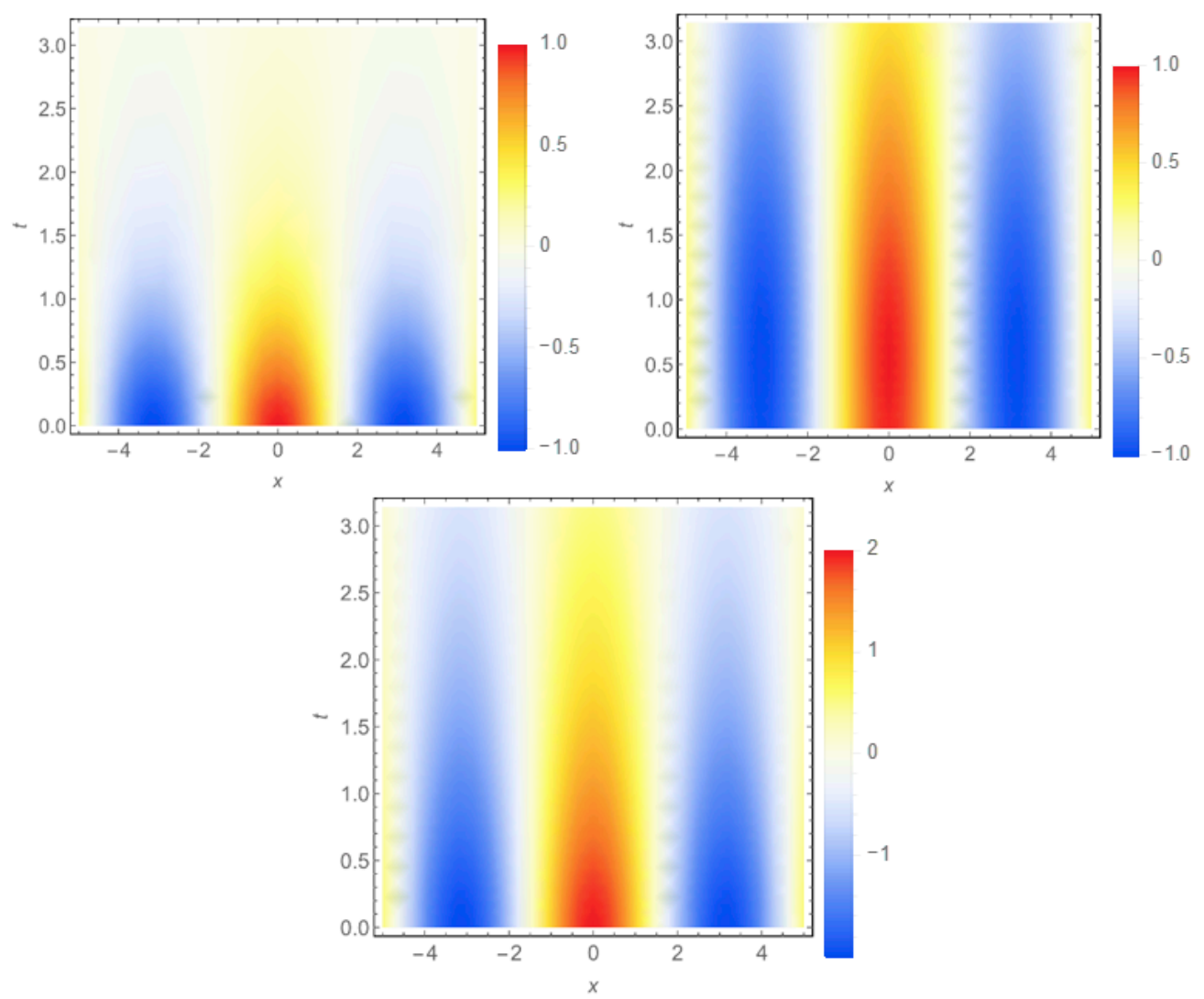

Figure 1. Evolution of quasi-temperature components in a thin stationary film with $K n_{b}=K n_{d}=1$ for $v=0$ : $\theta_{b}(x, t)$-top left plot, $\theta_{d}(x, t)$-top right plot and $\theta(x, t)=\theta_{b}(x, t)+\theta_{d}(x, t)$-bottom plot. The Cauchy conditions for PDEs (9) and (10) system are $\theta_{b}(x, 0)=\theta_{d}(x, 0)=e^{i n x}, \partial_{t} \theta_{b}(x, 0)=\partial_{t} \theta_{d}(x, 0)=0$.

In the case when the rate of change of temperature for a given position in a field depends both on the instantaneous rate of change of temperature at that location $(\partial / \partial t)$ as well as on the rate at which the temperature is convected to that location by the fluid motion, the behaviors of the solutions change significantly. The solutions for $K n_{d}=K n_{b}=1$ and $v=10$, are presented in Figure 2, for $v=-10$ in Figure 3 . 

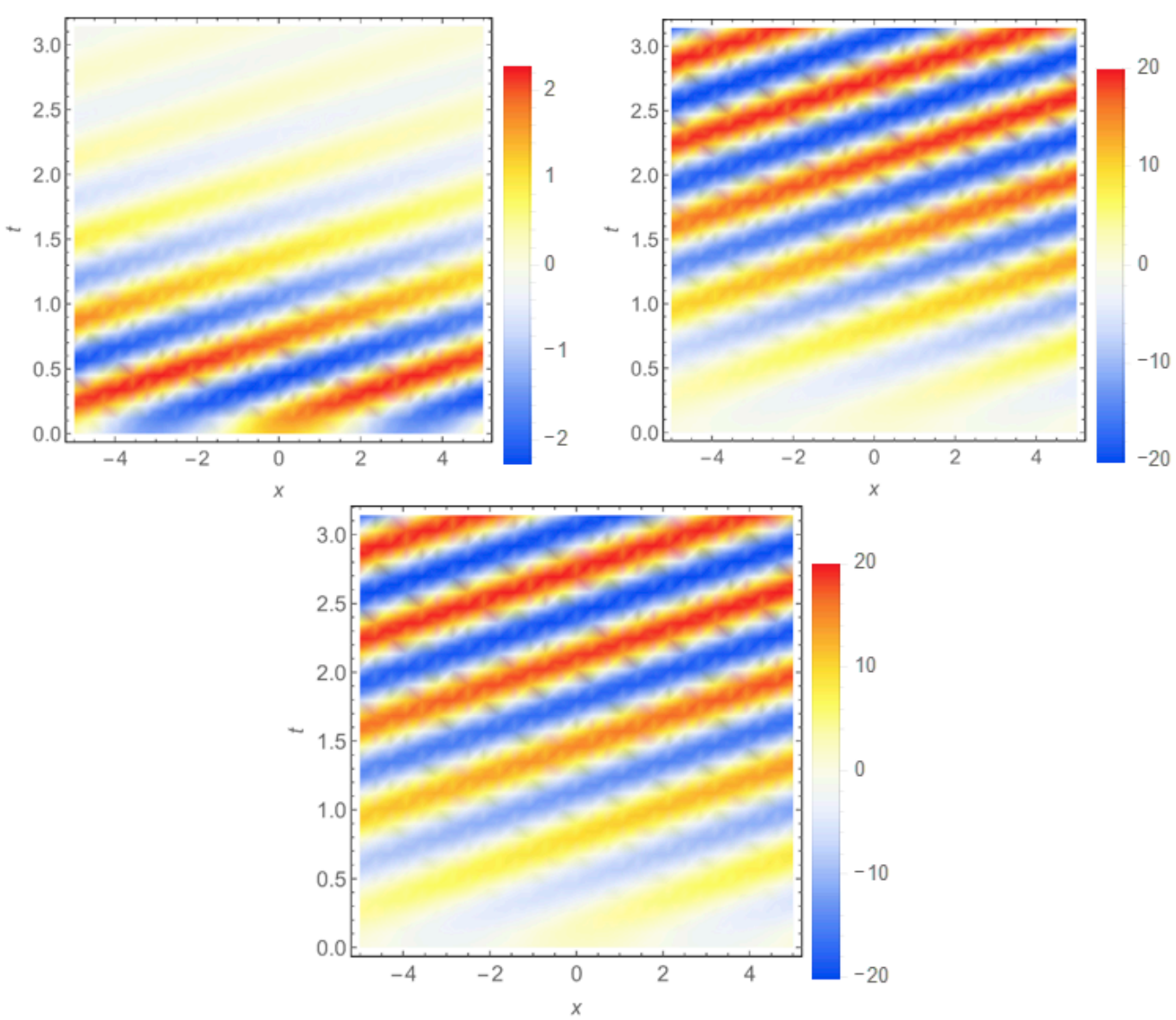

Figure 2. Evolution of quasi-temperature components in a thin film with $K n_{b}=K n_{d}=1$ for $v=+10$ : $\theta_{b}(x, t)$-top left plot, $\theta_{d}(x, t)$-top right plot and $\theta(x, t)=\theta_{b}(x, t)+\theta_{d}(x, t)$-bottom plot. The Cauchy conditions for PDEs (9) and (10) system are $\theta_{b}(x, 0)=\theta_{d}(x, 0)=e^{i n x}, \partial_{t} \theta_{b}(x, 0)=\partial_{t} \theta_{d}(x, 0)=0$.

The ballistic constituent $\theta_{b}$ rapidly vanishes with time; the direction of the colorful waves in the plot depends on the sign of the speed $v$ (see top left plots in Figures 2 and 3). Diffusive counterpart of the complete quasi-temperature $\theta_{d}$ has waves of increasing amplitude as seen in top right plots in Figures 2 and 3. The complete quasi-temperature $\theta=\theta_{d}+\theta_{b}$ has obvious non-Fourier behavior, which largely follows that of $\theta_{d}$.

For smaller values of Knudsen number, $K n=0.1$, the behavior of the harmonic solutions to ballistic heat propagation in thin films, obeying the Eqs. system (9) and (10) with Cauchy conditions (36) (37), has less distinctive wave interference with slower fade of the solution for $\theta_{b}$ and weaker amplitude increase speed for $\theta_{d}$; proper solutions for $v=+10$ are presented in Figure 4 .

It is important to note, that the whole system is symmetric under the speed inversion, which becomes obvious with account for formulae (38)-(44). 

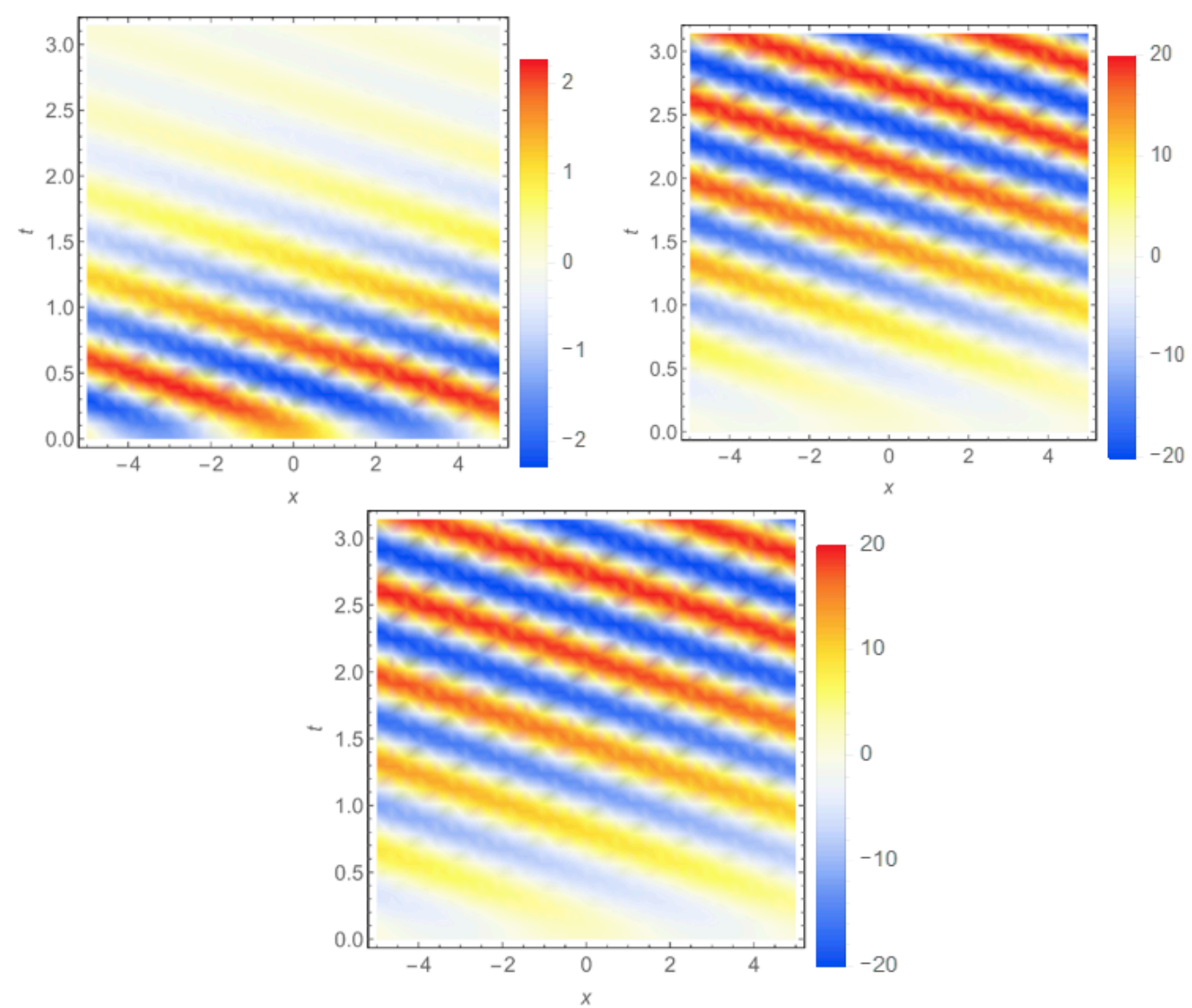

Figure 3. Evolution of quasi-temperature components in a thin film with $K n_{b}=K n_{d}=1$ for $v=-10$ : $\theta_{b}(x, t)$-top left plot, $\theta_{d}(x, t)$-top right plot and $\theta(x, t)=\theta_{b}(x, t)+\theta_{d}(x, t)$-bottom plot. The Cauchy conditions for PDEs (9) and (10) system are $\theta_{b}(x, 0)=\theta_{d}(x, 0)=e^{i n x}, \partial_{t} \theta_{b}(x, 0)=\partial_{t} \theta_{d}(x, 0)=0$.

Note, that for $K n_{b}=K n_{d}=0.1 \theta_{b}(x, t)$ and $\theta_{d}(x, t)$ constituents behave differently (see Figure 4). The diffusive constituent slowly grows as the ballistic constituent shows gradual amplitude decrease, as shown in Figure 5. Moreover, for $v=10$ the complete quasi-temperature $\theta(x, t)=\theta_{b}(x, t)+\theta_{d}(x, t)$ monotonously increases, following the behavior of the diffusive constituent as shown in Figure 5. The maximum principle, established though for parabolic equations, is violated for the diffusive component and complete quasi-temperature with Cauchy conditions for non-Fourier ballistic heat transport in thin films. In all of the cases, the evolution of $\theta_{b}(x, t)$ occurs faster than that of $\theta_{d}(x, t)$; the two-speed heat propagation process can be seen in every set of plots in Figures 1-5. 

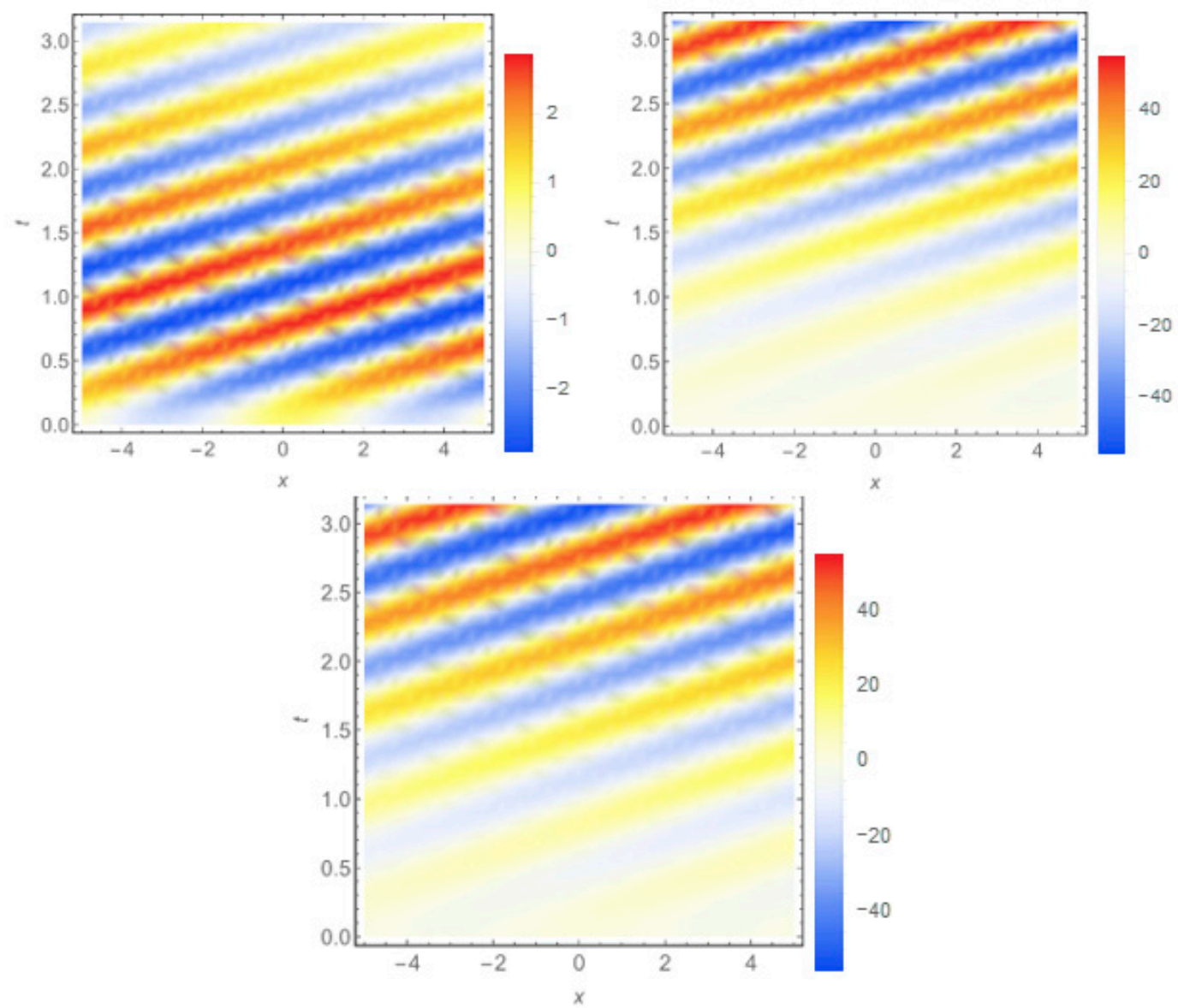

Figure 4. Evolution of quasi-temperature components in a thin film with $K n_{b}=K n_{d}=0.1$ for $v=+10$ : $\theta_{b}(x, t)$-top left plot, $\theta_{d}(x, t)$ —top right plot and $\theta(x, t)=\theta_{b}(x, t)+\theta_{d}(x, t)$-bottom plot. The Cauchy conditions for PDEs (9) and (10) system are $\theta_{b}(x, 0)=\theta_{d}(x, 0)=e^{i n x}, \partial_{t} \theta_{b}(x, 0)=\partial_{t} \theta_{d}(x, 0)=0$.
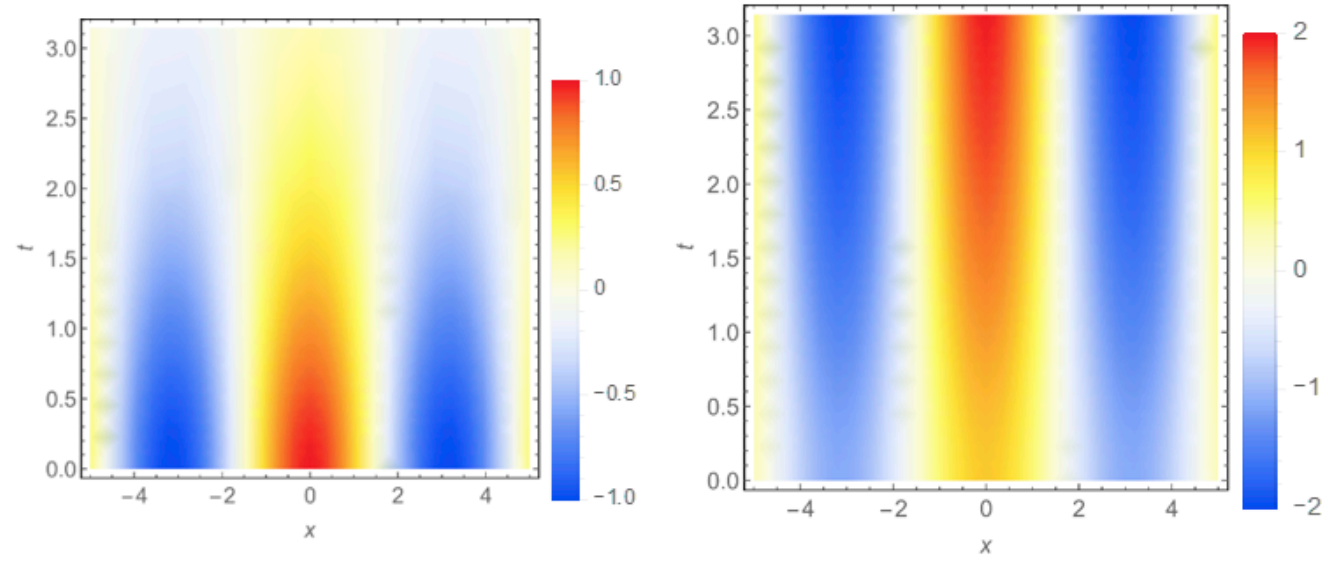

Figure 5. Evolution of quasi-temperature components in a stationary thin film with $K n_{b}=K n_{d}=0.1$ for $v=0: \theta_{b}(x, t)$-left plot, $\theta_{d}(x, t)$-right plot. The Cauchy conditions for PDEs (9) and (10) system are $\theta_{b}(x, 0)=\theta_{d}(x, 0)=e^{i n x}, \partial_{t} \theta_{b}(x, 0)=\partial_{t} \theta_{d}(x, 0)=0$.

The exploration of the solutions for Dirichlet conditions will be done elsewhere.

It should be noted that the one-dimensional energy balance equation yields zero value for the initial heat flux, if $B=-i n v A$. Above we have obtained the solutions, which allow nonzero initial conditions on the first time derivative; we have investigated some examples while writing this paper. 
For example, for the case of $K n_{b}=K n_{d}=1, v=10$ the maximum principle is violated for both zero and nonzero initial conditions imposed on the first time-derivative of the ballistic component; however, there was no significant qualitative difference in the behaviors of the diffusive component in this case.

Explicit study for the solution in thin films with zero initial heat flux and non-zero velocity $v$ will be done in forthcoming publications.

\section{Conclusions}

In the present work we have studied and analytically solved one-dimensional heat transport equations of Cattaneo- and Guyer-Krumhansl-type with substantial derivative. Exponential differential operators and the operational method were used to obtain integral forms of exact particular solutions to heat transport equations. The media speed is accounted for by the exponential differential operator $e^{-v t \partial_{x}}$, which produces a shift $x-v t$. Together with the heat operator $\hat{S}$ they transform the initial temperature profile and determine its evolution. Their action on the initial polynomial $\sum_{n} x^{n}$ yields sums of the Hermite polynomials $H_{n}(x, y)$; the action on the monomial-exponential function yields more complicated sums of Hermite polynomials. Proper exact operational solutions were obtained in the sums of converging integrals of elementary functions. Several examples were considered, the solutions for $F(x, 0)=x^{2}, F(x, 0)=x^{2} e^{-x}$ and $F(x, 0)=e^{i n x}$ were given. The solutions exactly satisfy the heat transport equations, which has been proven by direct substitution.

The equations with substantial derivative of Guyer-Krumhansl-type and of telegrapher's-type are demonstrated to have similar structure in the harmonic ansatz. Their exact harmonic solutions have been obtained by both operational method and the separation of variables. The latter reduces these PDEs to ODEs. We have shown that the harmonic solutions may have local and global extremums in the domain due to the interference of the Cattaneo heat waves; proper second-order time-derivative term also insures finite speed of the temperature perturbation propagation. We have shown that in a GK-type equation with dominant ballistic term $\delta$, the Cattaneo heat waves are damped.

We have obtained the exact analytical solution to the ballistic heat transport in thin films by solving the system of inhomogeneous PDEs [30] in the harmonic ansatz; both Cauchy and Dirichlet conditions were explored. We found that true ballistic constituent of quasi-temperature, $\theta_{b}$, evolves much faster than the diffusive counterpart of quasi-temperature, $\theta_{d}$. We have studied the evolution of the complete quasi-temperature $\theta(x, t)=\theta_{b}(x, t)+\theta_{d}(x, t)$ under the ballistic conditions; the latter apply when the phonon mean free path $l$ is comparable with the system scale $L$. The quasi-temperature $\theta$ first senses fast evolving true ballistic part $\theta_{b}$. The contribution of $\theta_{b}$ sets the r.h.s. in the inhomogeneous $\mathrm{DE}$ for the component $\theta_{d}$. After relatively fast relaxation of $\theta_{b}$, further evolution of the complete quasi-temperature $\theta$ follows the diffusive constituent $\theta_{d}$. Thus the initial fast-developing true ballistic component $\theta_{b}$ determines the evolution of the complete quasi-temperature of the system $\theta$ by imposing distribution for the diffusive quasi-temperature $\theta_{d}$ at the beginning of the process.

The obtained harmonic solutions for the Cauchy problem (9), (10), (36), (37) were applied for the study of the temperature patterns in thin films for the Knudsen number values $K n=0.1$ and $K n=1$. In the stationary case $v=0$, the true ballistic component $\theta_{b}$ monotonously relaxes (see Figures 1 and 5); its diffusive counterpart $\theta_{d}$ behavior shows noticeable dependence on the value of the Knudsen numbers: for $K n=1$ it slowly relaxes (see Figure 5) and for $K n=0.1$ it droningly grows (see Figure 1). The effect of the speed of the observer on the perceived heat transport in thin films is distinguished for the pure ballistic and diffusive quasi-temperature constituents. In the presence of the motion, the extremum of the ballistic component $\theta_{b}$ occurs inside the domain after the initial moment $t=0$ (see Figures 2-4). The diffusive quasi-temperature component monotonously grows in the domain both in the weak ballistic (see Figure 4) and strong ballistic cases (see Figures 2 and 3). This behavior contrasts common Fourier heat diffusion, for which the maximum principle holds. It is also noticeable that the system shows symmetric behavior while the value of the speed is reversed, which does not contradict the Second law. The Knudsen number variation from 0.1 to 1 in the presence of the 
speed $v=10$, are practically not sensed by the system. Thus the speed of the observer $v$ influences the registered temperature rather than the ballistic heat transport contribution.

Author Contributions: K.Z. has made the major work on the paper. N.G. has made a contribution in the Section 3. D.O. has made the contribution in Sections 4 and 5.

Acknowledgments: The authors are grateful to Anatolii Borisov, Andrey Lobanov for the discussions of the heat equations; to Laszlo I. Kiss (UQAC, Department of Applied Sciences, Quebec University) and Yasar Demirel (Department of Chemical and Biological Engineering, University of Nebraska, Lincoln) for discussions of the heat evolution patterns.

Conflicts of Interest: The authors declare no conflict of interest.

\section{References}

1. Fourier, J.P.J. The Analytical Theory of Heat; Cambridge University Press: London, UK, 1878.

2. Ackerman, C.C.; Guyer, R.A. Temperature pulses in dielectric solids. Ann. Phys. 1968, 50, 128-185. [CrossRef]

3. Ackerman, C.C.; Overton, W.C. Second sound in solid helium-3. Phys. Rev. Lett. 1969, 22, 764. [CrossRef]

4. McNelly, T.F.; Rogers, S.J.; Channin, D.J.; Rollefson, R.; Goubau, W.M.; Schmidt, G.E.; Krumhansl, J.A.; Pohl, R.O. Heat pulses in NaF: Onset of second sound. Phys. Rev. Lett. 1970, 24, 100. [CrossRef]

5. Narayanamurti, V.; Dynes, R.D. Observation of second sound in Bismuth. Phys. Rev. Lett. 1972, 26, 1461-1465. [CrossRef]

6. Peshkov, V. Second sound in Helium II. J. Phys. (Moscow) 1944, 8, 381.

7. Cattaneo, C. Sur une forme de l'equation de la chaleur eliminant le paradoxe d'une propagation instantanee. C. R. l'Acad. Sci. Paris 1958, 247, 431-433.

8. Terman, F.E. Radio Engineers' Handbook, 1st ed.; McGraw-Hill: New York, NY, USA, 1943.

9. Both, S.; Czél, B.; Fülöp, T.; Ván, P.; Verhás, J. Deviation from the Fourier law in room-temperature heat pulse experiments. J. Non-Equilib. Thermodyn. 2016, 41, 41-48. [CrossRef]

10. Kovács, R.; Ván, P. Models of ballistic propagation of heat at low temperatures. Int. J. Thermophys. 2016, $37,95$. [CrossRef]

11. Ván, P.; Berezovski, A.; Fülöp, T.; Gróf, G.; Kovács, R.; Lovas, Á.; Verhás, J. Guyer-Krumhansl-type heat conduction at room temperature. EPL 2017, 118, 50005. [CrossRef]

12. Cahill, D.G. Thermal conductivity measurement from 30 to $750 \mathrm{~K}$ : The $3 \omega$ method. Rev. Sci. Instrum. 1990, 61, 802-808. [CrossRef]

13. Tang, D.W.; Araki, N. Non-Fourier heat conduction behaviour in finite mediums under pulse surface heating. Mater. Sci. Eng. A 2000, 292, 173-178. [CrossRef]

14. Kaminski, W. Hyperbolic heat conduction equations for materials with a nonhomogeneous inner structure. J. Heat Transf. 1990, 112, 555-560. [CrossRef]

15. Mitra, K.; Kumar, S.; Vedavarz, A.; Moallemi, M.K. Experimental evidence of hyperbolic heat conduction in processed meat. J. Heat Transf. 1995, 117, 568-573. [CrossRef]

16. Herwig, H.; Beckert, K. Fourier versus non-Fourier heat conduction in materials with a nonhomogeneous inner structure. J. Heat Transf. 2000, 122, 363-365. [CrossRef]

17. Roetzel, W.; Putra, N.; Das, S.K. Experiment and analysis for non-Fourier conduction in materials with non-homogeneous inner structure. Int. J. Therm. Sci. 2003, 42, 541-552. [CrossRef]

18. Sazhin, S.S. Modelling of fuel droplet heating and evaporation: Recent results and unsolved problems. Fuel 2017, 196, 69-101. [CrossRef]

19. Scott, E.P.; Tilahun, M.; Vick, B. The question of thermal waves in heterogeneous and biological materials. J. Biomech. Eng. 2009, 131, 074518. [CrossRef] [PubMed]

20. Ricciu, R.; Besalduch, L.A.; Galatioto, A.; Ciulla, G. Thermal characterization of insulating materials. Renew. Sustain. Energy Rev. 2018, 82, 1765-1773. [CrossRef]

21. Porrà, J.M.; Masoliver, J.; Weiss, G.H. When the telegrapher's equation furnishes a better approximation to the transport equation than the diffusion approximation. Phys. Rev. E 1997, 55, 7771-7774. [CrossRef]

22. Shiomi, J.; Maruyama, S. Non-Fourier heat conduction in a single-walled carbon nanotube: Classical molecular dynamics simulations. Phys. Rev. B 2006, 73, 205420. [CrossRef] 
23. Baringhaus, J.; Ruan, M.; Edler, F.; Tejeda, A.; Sicot, M.; Taleb-Ibrahimi, A.; Li, A.P.; Jiang, Z.; Conrad, E.H.; Berger, C.; et al. Exceptional ballistic transport in epitaxial graphene nanoribbons. Nature 2014, 506, 349-354. [CrossRef] [PubMed]

24. Hochbaum, A.I.; Chen, R.; Delgado, R.D.; Liang, W.; Garnett, E.C.; Najarian, M.; Majumdar, A.; Yang, P. Enhanced thermoelectric performance of rough silicon nanowires. Nature (London) 2008, 451, 163-167. [CrossRef] [PubMed]

25. Boukai, A.I.; Bunimovich, Y.; Tahir-Kheli, J.; Yu, J.-K.; Goddard, W.A.; Heath, J.R. Silicon nanowires as efficient thermoelectric materials. Nature (London) 2008, 451, 168. [CrossRef] [PubMed]

26. Paddock, C.A.; Eesley, G.L. Transient thermoreflectance from thin metal films. J. Appl. Phys. 1986, 60, $285-290$. [CrossRef]

27. Maldovan, M. Transition between ballistic and diffusive heat transport regimes in silicon materials. Appl. Phys. Lett. 2012, 101, 113110. [CrossRef]

28. Guyer, R.A.; Krumhansl, J.A. Solution of the linearized phonon Boltzmann equation. Phys. Rev. 1966, 148, 766-778. [CrossRef]

29. Guyer, R.A.; Krumhansl, J.A. Thermal conductivity, second sound and phonon hydrodynamic phenomena in non-metallic crystals. Phys. Rev. 1966, 148, 778-788. [CrossRef]

30. Lebon, G.; Machrafi, H.; Gremela, M.; Dubois, C. An extended thermodynamic model of transient heat conduction at sub-continuum scales. Proc. R. Soc. A 2011, 467, 3241-3256. [CrossRef]

31. Müller, I.; Ruggeri, T. Rational Extended Thermodynamics; Springer: Berlin/Heidelberg, Germany, 1998.

32. Rogolino, P.; Kovács, R.; Ván, P.; Cimmelli, V.A. Generalized heat-transport equations: Parabolic and hyperbolic models. Contin. Mech. Thermodyn. 2018. [CrossRef]

33. Moosaie, A. Non-Fourier heat conduction in a finite medium with insulated boundaries and arbitrary initial conditions. Int. Commun. Heat Mass Transf. 2008, 35, 103-111. [CrossRef]

34. Ahmadikia1, H.; Rismanian, M. Analytical solution of non-Fourier heat conduction problem on a fin under periodic boundary conditions. J. Mech. Sci. Technol. 2011, 25, 2919-2926. [CrossRef]

35. Yen, C.C.; Wu, C.Y. Modelling hyperbolic heat conduction in a finite medium with periodic thermal disturbance and surface radiation. Appl. Math. Model. 2003, 27, 397-408. [CrossRef]

36. Lewandowska, M. Hyperbolic heat conduction in the semi-infinite body with a time-dependent laser heat source. Heat Mass Transf. 2001, 37, 333-342. [CrossRef]

37. Lewandowska, M.; Malinowski, L. An analytical solution of the hyperbolic heat conduction equation for the case of a finite medium symmetrically heated on both sides. Int. Commun. Heat Mass Transf. 2006, 33, 61-69. [CrossRef]

38. Saedodin, S.; Torabi, M. Analytical solution of non-Fourier heat conduction in cylindrical coordinates. Int. Rev. Mech. Eng. 2009, 3, 726-732.

39. Challamel, N.; Grazide, C.; Picandet, V.; Perrot, A.; Zhang, Y. A nonlocal Fourier's law and its application to the heat conduction of one-dimensional and two-dimensional thermal lattices. C. R. Mec. 2016, 344, 388-401. [CrossRef]

40. Saedodin, S.; Torabi, M. Algebraically explicit analytical solution of three-dimensional hyperbolic heat conduction equation. Adv. Theor. Appl. Mech. 2010, 3, 369-383.

41. Chen, G. Ballistic-diffusive heat-conduction equations. Phys. Rev. Lett. 2001, 86, 2297-2300. [CrossRef] [PubMed]

42. Hsiao, T.K.; Chang, H.K.; Liou, S.C.; Chu, M.W.; Lee, S.C.; Chang, C.W. Observation of room-temperature ballistic thermal conduction persisting over $8.3 \mathrm{~mm}$ in SiGe nanowires. Nat. Nanotechnol. 2013, 8, 534-538. [CrossRef] [PubMed]

43. Zhang, Y.; Ye, W. Modified ballistic-diffusive equations for transient non-continuum heat conduction. Int. J. Heat Mass Transf. 2015, 83, 51-63. [CrossRef]

44. Kovacs, R.; Van, P. Generalized heat conduction in heat pulse experiments. Int. J. Heat Mass Transf. 2015, 83, 613-620. [CrossRef]

45. KZhukovsky, V. Operational method of solution of linear non-integer ordinary and partial differential equations. SpringerPlus 2016, 5, 119. [CrossRef] [PubMed]

46. Zhukovsky, K. Operational approach and solutions of hyperbolic heat conduction equations. Axioms 2016, 5, 28. [CrossRef] 
47. Zhukovsky, K.V.; Srivastava, H.M. Analytical solutions for heat diffusion beyond Fourier law. Appl. Math. Comput. 2017, 293, 423-437. [CrossRef]

48. Zhukovsky, K.V. Violation of the maximum principle and negative solutions with pulse propagation in Guyer-Krumhansl model. Int. J. Heat Mass Transf. 2016, 98, 523-529. [CrossRef]

49. Zhukovsky, K.V. Exact solution of Guyer-Krumhansl type heat equation by operational method. Int. J. Heat Mass Transf. 2016, 96, 132-144. [CrossRef]

50. Zhukovsky, K. Exact negative solutions for Guyer-Krumhansl type equation and the violation of the maximum principle. Entropy 2017, 19, 440. [CrossRef]

51. Zhukovsky, K. Operational solution for some types of second order differential equations and for relevant physical problems. J. Math. Anal. Appl. 2017, 446, 628-647. [CrossRef]

52. Zhukovsky, K.V. A method of inverse differential operators using ortogonal polynomials and special functions for solving some types of differential equations and physical problems. Mosc. Univ. Phys. Bull. 2015, 70, 93-100. [CrossRef]

53. Zhukovsky, K. Solution of some types of differential equations: Operational calculus and inverse differential operators. Sci. World J. 2014, 2014. [CrossRef] [PubMed]

54. Zhukovsky, K.V. Solving evolutionary-type differential equations and physical problems using the operator method. Theor. Math. Phys. 2017, 190, 52-68. [CrossRef]

55. Dattoli, G.; Srivastava, H.M.; Zhukovsky, K.V. Operational methods and Differential Equations with applications to initial-value problems. Appl. Math. Comput. 2007, 184, 979-1001. [CrossRef]

56. Zhukovsky, K.V. Operational solution of differential equations with derivatives of non-integer order, Black-Scholes type and heat conduction. Mosc. Univ. Phys. Bull. 2016, 71, 237-244. [CrossRef]

57. Dattoli, G.; Srivastava, H.M.; Zhukovsky, K.V. Orthogonality properties of the Hermite and related polynomials. J. Comput. Appl. Math. 2005, 182, 165-172. [CrossRef]

58. Dattoli, G.; Srivastava, H.M.; Zhukovsky, K.V. A new family of integral transforms and their applications. Integral Transforms Spec. Funct. 2006, 17, 31-37. [CrossRef]

59. Srivastava, H.M.; Manocha, H.L. A Treatise on Generating Functions; Halsted Press (Ellis Horwood Limited): Chichester, UK; John Wiley and Sons: New York, NY, USA; Chichester, UK; Brisbane, Australia; Toronto, ON, Canada, 1984.

60. Boucetta, A.; Ghodbane, H.; Ayad, M.Y.; Bahri, M. A review on the performance and modelling of proton exchange membrane fuel cells. AIP Conf. Proc. 2016, 1758, 030019.

61. Arato, E.; Pinna, M.; Mazzoccoli, M.; Bosio, B. Gas-phase mass-transfer resistances at polymeric electrolyte membrane fuel cells electrodes: Theoretical analysis on the effectiveness of interdigitated and serpentine flow arrangements. Energies 2016, 9, 229. [CrossRef]

62. Maidhily, M.; Rajalakshmi, N.; Dhathathreyan, K.S. Electrochemical impedance spectroscopy as a diagnostic tool for the evaluation of flow field geometry in polymer electrolyte membrane fuel cells. Renew. Energy 2013, 51, $79-84$. [CrossRef]

63. St-Pierre, J. Hydrogen mass transport in fuel cell gas diffusion electrodes. Fuel Cells 2011, 11, $263-273$. [CrossRef]

64. Zhukovsky, K.; Pozio, A. Maximum current limitations of the PEM fuel cell with serpentine gas supply channels. J. Power Sources 2004, 130, 95-105. [CrossRef]

65. Zhukovsky, K.V. Three Dimensional model of gas transport in a porous diffuser of a polymer electrolyte fuel cell. AIChE J. 2003, 49, 3029-3036. [CrossRef]

66. Zhukovsky, K. Modeling of the Current Limitations of PEFC. AIChE J. 2006, 52, 2356-2366. [CrossRef]

67. Weber, A.Z.; Newman, J. Modeling transport in polymer-electrolyte fuel cells. Chem. Rev. 2004, 104, 4679-4726. [CrossRef] [PubMed]

68. Kawase, M.; Sato, K.; Mitsui, R.; Asonuma, H.; Kageyama, M.; Yamaguchi, K.; Inoue, G. Electrochemical reaction engineering of polymer electrolyte fuel cell. AIChE J. 2017, 63, 249-256. [CrossRef]

69. Rogié, B.; Monier-Vinard, E.; Nguyen, M.-N.; Bissuel, V.; Laraqi, N. Practical analytical modeling of 3D multi-layer Printed Wired Board with buried volumetric heating sources. Int. J. Therm. Sci. 2018, 129, 404-415. [CrossRef]

70. Calvo-Schwarzwälder, M.; Hennessy, M.G.; Torres, P.; Myers, T.G.; Alvarez, F.X. A slip-based model for the size-dependent effective thermal conductivity of nanowires. Int. Commun. Heat Mass Transf. 2018, 91, 57-63. [CrossRef]

71. Zhukovskij, K.V. Gas flow in long microchannels. Vestn. Mosk. Univ. Ser. 3 Fiz. Astron. 2001, 3, 49-54. 
72. Zhukovsky, K.V. A harmonic solution for the hyperbolic heat conduction equation and its relationship to the Guyer-Krumhansl Equation. Mosc. Univ. Phys. Bull. 2018, 73, 45-52. [CrossRef]

73. Zhukovsky, K. Exact harmonic solution to ballistic type heat propagation in thin films and wires. Int. J. Heat Mass Transf. 2018, 120, 944-955. [CrossRef]

74. Zhukovsky, K.; Oskolkov, D. Exact harmonic solutions to Guyer-Krumhansl-type equation and application to heat transport in thin films. Contin. Mech. Thermodyn. 2018. [CrossRef]

75. Ali, Y.M.; Zhang, L.C. Relativistic heat conduction. Int. J. Heat Mass Transf. 2005, 48, 2397-2406. [CrossRef]

76. Al-Khairy, R.T.; Al-Ofey, Z.M. Analytical solution of the hyperbolic heat conduction equation for moving semi-infinite medium under the effect of time-dependent laser heat source. J. Appl. Math. 2009, 2009. [CrossRef]

77. Wolf, K.B. Integral Transforms in Science and Engineering; New York Plenum Press: New York, NY, USA, 1979.

(c) 2018 by the authors. Licensee MDPI, Basel, Switzerland. This article is an open access article distributed under the terms and conditions of the Creative Commons Attribution (CC BY) license (http://creativecommons.org/licenses/by/4.0/). 